\title{
Acquired Gerbode defect following endocarditis of the tricuspid valve: a case report and literature review
}

\author{
Edvin Prifti ${ }^{1}$, Fadil Ademaj ${ }^{2 *}$, Arben Baboci ${ }^{3}$ and Aurel Demiraj ${ }^{2}$
}

\begin{abstract}
The Gerbode's defect is a communication between the left ventricle and right atrium. It is usually congenital, but rarely is acquired, as a complication of endocarditis, myocardial infarction, trauma, or after previous cardiac surgery. The acquired Gerbode defect with involvement of the tricuspid valve acquired after bacterial endocarditis can be challenging to repair. We present a rare case of young woman, with endocarditis of the tricuspid valve and acquired Gerbode defect without previous cardiac surgery. She underwent successful surgical closure of the Gerbode defect and reconstruction of the septal leaflet of the tricuspid valve using a an autologous pericardial patch. A total of 20 other cases were reported with acquired Gerbode defect due to endocarditis in patients without previous cardiac surgery. Three other cases presented acquired Gerbode defect due to myocardial infarction and two due to chest trauma. Another series of 62 patients presented acquired Gerbode defect after previous cardiac surgery. Surgical treatment is always feasible with excellent outcome. However the percutanous transcatheter closure remains an excellent option especially in high risk patients.
\end{abstract}

Keywords: Acquired, Gerbode, defect

\section{Introduction}

The communication between the left ventricle and right atrium was firstly reported in 1838 by Thurman [1]. In 1957, Gerbode et al. [2] reported the first 5 cases with such a heart defect undergoing successful surgical repair. Such a defect is usually congenital, but rarely is acquired, as a complication of endocarditis [3], myocardial infarction, blunt chest trauma or after previous cardiac surgery [4]. This can be anatomically possible because the normal tricuspid valve is more apically displaced than the mitral valve. Acquired Gerbode defects with large septal destructions and vegetations involving the tricuspid valve can be challenging and might require complex patch repair. We present a case of our patient with this uncommon complication of endocarditis, simulating severe pulmonary hypertension.

\footnotetext{
* Correspondence: fadilademaj1971@gmail.com

${ }^{2}$ Division of Cardiology, Regional Hospital of Gjakovo, Gjakovo, Kosovo Full list of author information is available at the end of the article
}

\section{Case report}

A 40 year old lady from Kosovo, was referred to our hospital for severe pulmonary arterial hypertension and a mass in right atrium suspected for vegetation. About one month before, she was admitted in another hospital and received iv medication. The patient was febrile and the C-reactive protein, white cell count and erythrocyte sedimentation rate were elevated. Blood cultures demonstrated a methacilin sensitive Staphylococcus aureus growth.

Transthoracic echocardiograhy demonstrated a mobile, irregularly shaped, oscillating and highly mobile mass, located above the tricuspid valve septal leaflet (Fig. 1b). A clear jet across a small defect between left ventricle and right atrium consistent with Gerbode type defect was identified. The direction of the Doppler signal also leads to the true diagnosis (Fig. 1a). Cardiac magnetic resonance demonstrated a supravalvular flow associated with infravalvular jet according to the type $\mathrm{C}$ acquired Gerbode defect (Fig. 1c and 1d). A normal lung scan excluded pulmonary embolism. The tricuspid regurgitation was considered mild- to- moderate 


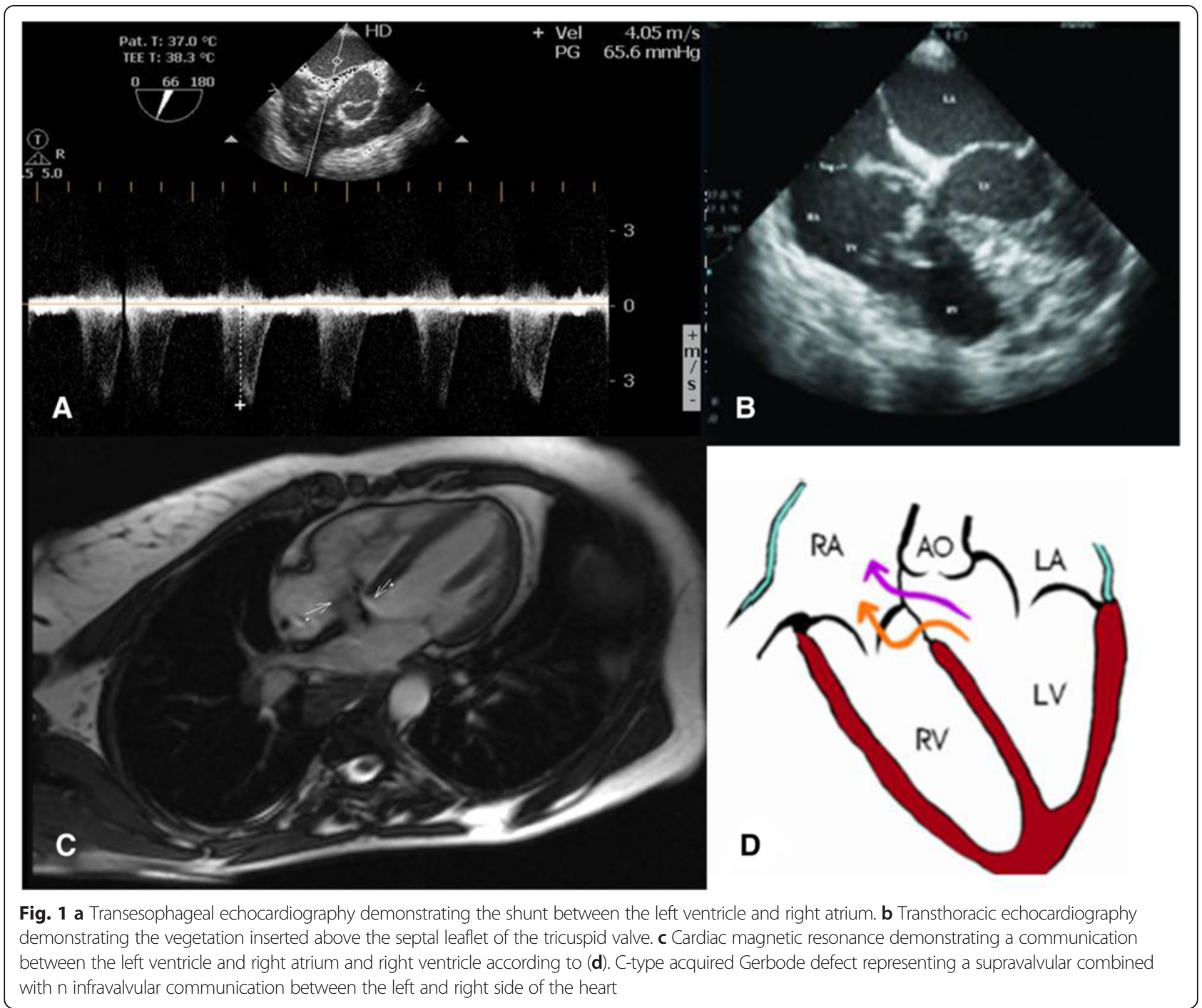

with estimated pulmonary arterial systolic pressure about 60-80 mmHg.

The patients underwent surgery after 2 weeks of antiobiotic therapy. Through a right atriotomy, large vegetation was attached to the septal leaflet and anterior leaflet of tricuspid valve was identified. On removal of the vegetation, a defect was found communicating between the left ventricle and right atrium (Fig. 2a and 2b). This defect represented an acquired Gerbode defect and was closed by two $5 / 0$ pledgeted prolene sutures (Fig. 2c). Then the septal leaflet of tricuspid valve was resected and was replaced with a trimmed autologous pericardial patch. Anteriorly the newly created septal leaflet was attached to the anterior leaflet. Then, two synthetic chorda were employed (Fig. 2d). The hydraulic maneuver demonstrated trivial tricuspid valve regurgitation (Fig. 2c). Then the right atrium was closed. After an uneventfully post-operative period, the patient was discharged home in good clinical condition. Echocardiogram demonstrated trivial tricuspid valve regurgitation and no residual shunt. One year later the patient was doing well. The transthoracic echocardiography at follow-up demonstrated a moderate tricuspid valve regurgitation and no residual shunt.

\section{Comment}

Gerbode described such a defect as a congenital atrioventricular shunt originating from the interventricular membranous septum with regurgitation into the right atrium through a defect or cleft in the tricuspid valve leaflet [2]. Less common is the acquired form of a Gerbode defect, which is often associated with bacterial endocarditis [5-24], myocardial infarction [25-27], blunt chest trauma [28, 29] or post previous cardiac surgical procedures [30,31].

After a careful revision of the literature we found 25 other reported cases with acquired Gerbode defect without previous cardiac surgery. In 22 of them, including our case, the cause was endocarditis. Only 4 patients were females. 7 out 21 cases presented endocarditis due 

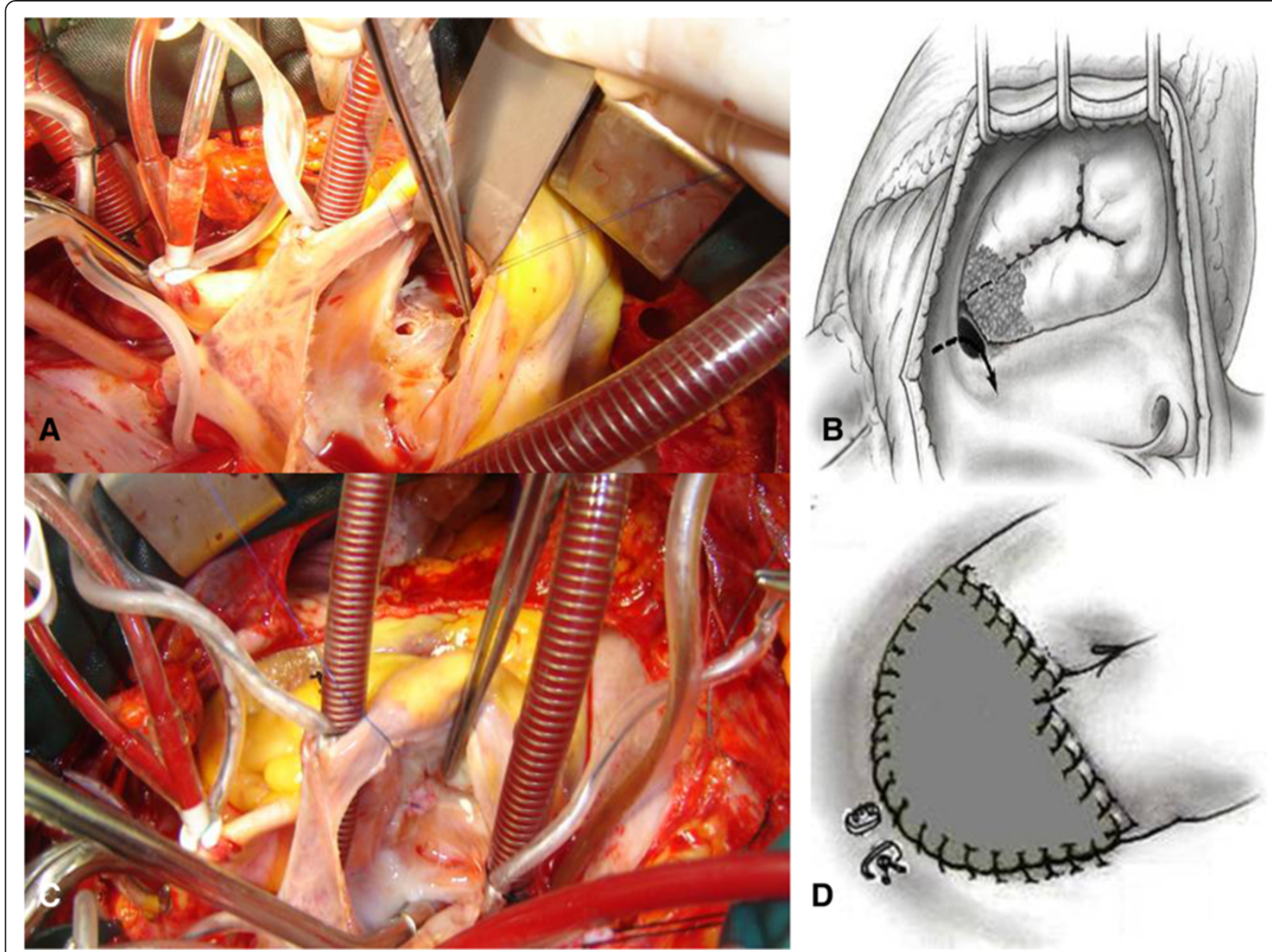

Fig. 2 a Intraoperative view demonstrating the acquired Gerbode defect after removing the septal leaflet and part of the anterior leaflet of the tricuspid valve. $\mathbf{b}$ A diagram representing the extension of the destructed valvular tissue. $\mathbf{c}$ Hydraulic maneuver after closure of the acquired Gerbode defect and reconstruction of the septal leaflet of the tricuspid valve. $\mathbf{d}$ A diagram demonstrating the final view of the operation

to Staphylococcus aureus, usually involving the aortic valve extending below the aortic annulus onto the upper part of the interventricular septum. Infective tissue destruction leads to a perforation of the septum creating a communication between the left ventricle and the right atrium. However 8 out of 21 cases including our case presented tricuspid valve endocarditis causing an acquired Gerbode defect (Table 1). In difference to the endocarditis of the left side, in the tricuspid valve endocarditis the vegetations and destructed tissue are located in the right side so, it might be more than enough the closure of the communication only on the right side, if healthy tissue is present as in our case. The mortality was almost $9 \%$ in patients with endocarditis. Also the postoperative complications such as renal failure was identified in 3 patients (13.6\%) and complete atrioventricular block in 3 patients (13.6 \%). The high incidence of the complete atrioventricularf block might be explained with the closed vicinity of the Gerbode defect with the conduction system and atrioventricular node.
Interestingely in none of the cases with Gerbode defect without prior cardiac surgery undergoing surgical correction is reported recurrence of the communication between the left ventricle and right atrium or endocarditis recurrence.

Three other cases acquired Gerbode defect post myocardial infarction were found in the literature and all of them presented inferior myocardial infarction. 2 of them died after surgery. Two other patients were found with acquired Gerbode defect due to blunt chest trauma or bullet penetration. The overall mortality in 26 patients without prior cardiac surgery was $15.4 \%$. The postoperative hospital stay was less than 2 weeks in the survived cases.

Interestingely, acquired Gerbode defect after previous cardiac surgery was found in 62 other patients (Table 2). 26 of them underwent surgical closure of the defect and 18 percutaneous closure employing different occlude devices. 11 patients did not undergo any interventional procedure, probably due to small shunt or high operative 
Table 1 Patients with acquired Gerbode defect without prior cardiac surgery

\begin{tabular}{|c|c|c|c|c|c|c|c|}
\hline Author (Ref) & Year & Gender/Age & Location & Bacteria & Diagnosis & Treatment & Outcome \\
\hline 1. Battin [5] & 1991 & Male/15 & na & na & TTE & Surgery & Survived \\
\hline 2. Saiki [6] & 1994 & Male/42 & $\mathrm{MV}, \mathrm{AV}$ & Streptococcus hemolyticus & TTE, & Surgery & Survived \\
\hline 3. Katoh [7] & 1994 & Male/58 & TV & na & na & Surgery & Survived \\
\hline 4. Elian [8] & 1995 & Male/64 & TV & Staphylococcus aureus & TTE, TEE, CC & Surgery & Survived \\
\hline 5. Velebit [9] & 1995 & Male/ 30 & BAV & Staphylococcus aureus & TEE, CC & Surgery & Survived(AVB) \\
\hline 6. Winslow [10] & 1995 & Male/ 30 & AV & Staphylococcus aureus & TTE, TEE & Surgery & Survived \\
\hline 7. Michel [11] & 1996 & Male/52 & AV & Streptococcus viridans & TTE, TEE & Conservative & Survived \\
\hline 8. Alphonso [12] & 2003 & Male/ 63 & $\mathrm{AV}$ & Culture negative & TTE & Surgery & Survived \\
\hline 9. Raja [13] & 2006 & Male/47 & RA & Staphylococcus aureus & TTE, TEE & Surgery & Survived(RF) \\
\hline 10. Fukui [14] & 2007 & Male/57 & $\mathrm{TV}, \mathrm{AV}, \mathrm{MV}$ & na & TEE & Surgery & Survived \\
\hline 11. Tatewaki [15] & 2008 & Female/7 & TV, AV, MV & Staphylococcus aureus & TEE, CT & Surgery & Survived \\
\hline 12. Inouel [16] & 2009 & Female/21 & $\mathrm{AV}$ & Culture negative & TTE, TEE & Surgery & Survived \\
\hline 13. Cortez-Dias [17] & 2009 & Male/59 & MV & Staphylococcus aureus & TTE, TEE & Conservative & $\operatorname{Died}(A \vee B, R F)$ \\
\hline 14. Mendoza [18] & 2009 & Female/52 & AV & Streptococcus mutans & TTE, $\mathrm{CT}$ & Surgery & Survived \\
\hline 15. Hori [19] & 2010 & Male/41 & BAV & na & TTE & Surgery & Survived \\
\hline 16. Matt [20] & 2010 & Male/35 & AV & Hemophilus aphrophilus & TTE,TEE & Surgery & Survived(AVB) \\
\hline 17. Ota [21] & 2011 & Male/71 & AV & Streptococcus pneumonia & TTE,TEE & Surgery & Survived \\
\hline 18. Pillai [22] & 2011 & Male/12 & TV & Culture negative & TEE & Surgery & Survived \\
\hline 19. Carpenter [23] & 2012 & Male/22 & TV & Staphylococcus lugdunensis & TEE, CT & Surgery & Survived \\
\hline 20. Hsu [24] & 2014 & Male/40 & BAV & Cardiobacterium hominis & $\mathrm{TEE}$ & Surgery & Died(RF) \\
\hline 21. Prifti et al. & 2015 & Female/40 & TV & Staphylococcus aureus & TTE, TEE & Surgery & Survived \\
\hline & & & \multicolumn{5}{|l|}{ Area of myocardial infarction } \\
\hline 22. Hole [25] & 1995 & Male/63 & \multicolumn{2}{|l|}{ Inferior myocardial infarction } & TTE & Surgery & Survived \\
\hline 23. Jobic [26] & 1997 & Female/72 & \multicolumn{2}{|l|}{ Inferior myocardial infarction } & TTE, TEE & Surgery & Died (RF) \\
\hline 24. Newman [27] & 1996 & Male/72 & \multicolumn{2}{|c|}{ Inferior myocardial infarction Trauma } & TTE, TEE & Surgery & Died \\
\hline 25. Venkatesh [28] & 1996 & Male/16 & \multicolumn{2}{|l|}{ Blunt trauma } & TTE, TEE & Surgery & Survived \\
\hline 26. Selinger [29] & 1998 & Male/70 & \multicolumn{2}{|l|}{ Bullet, trauma } & TTE,TEE,CC & Surgery & Survived \\
\hline
\end{tabular}

Legend: TTE Transthoracic echocardiography, TEE Transesophageal echocardiography, CC Cardiac catheterization, CT Cardiac tomography, na not available, AV Aortic valve, BAV Bicuspid Aortic Valve, MV Mitral valve, TV Tricuspid valve, RF Renal Failure, AVB Complete atrioventricular block

risk. Most of the patients were undergone previously aortic valve surgery or mitral valve surgery. However the mortality, in this group of patients despite all of them were redo operations, was almost $3.2 \%$ extremely lower than patients undergoing first time cardiac surgical procedure (Table 1).

The diagnosis was made in most of the cases by transthoracic and transesophageal echocardiography. It seems that echocardiographic examination is the most frequently diagnostic tool employed in these patients. Identification of an actual communication is often extremely difficult, so a careful and meticulous echocardiogram should be done in order to prevent echocardiographic misinterpretation of this defect as pulmonary arterial hypertension. The large systolic pressure gradient between the left ventricle and the right atrium would expectedly result in a high velocity systolic Doppler flow signal in right atrium and it can be sometimes mistakably diagnosed as tricuspid regurgitant jet simulating pulmonary arterial hypertension. However cardiac catheterization, cardiac tomography or magnetic resonance such as in our case offers valuable information. Interestingely our case after been diagnosed with Gerbode defect underwent cardiac magnetic resonance which revealed a class $C$ acquired Gerbode defect as previously described [4].

Treatment of the acquired Gerbode defect depends on symptoms, magnitude of shunt, flow volume, concomitant anatomic abnormalities and co-morbidities. Asymptomatic, chronic, small defects can be managed conservatively.

Percutaneous transcatheter closure techniques have been employed in almost $25 \%$ of patients, mostly in high risk surgical candidates due to previous valve replacement, advanced age, anti-coagulation, and multiple comorbidities. Advanced cardiac imaging techniques such 
Table 2 Patients with acquired Gerbode defect undergoing previous cardiac surgery

\begin{tabular}{|c|c|c|c|c|c|c|c|}
\hline Author & Year & Gender & Age & Diagnostic tool & Previous procedure & Treatment & Outcome \\
\hline 1. Katta et al. & 1994 & Male & 54 & TTE,TEE & Endomyocardial biopsy & Conservative & Survived \\
\hline 2. Dzwonczyk et al. & 1995 & Male & 25 & TTE & ASD repair & na & na \\
\hline 3. Dzwonczyk et al. & 1995 & Female & 72 & TTE & AVR, VSD repair & na & na \\
\hline 4. Fukui et al. & 2000 & Male & 53 & TEE & MVR $\times 2$ & Surgery & Survived \\
\hline 5. Benisty et al. & 2000 & Male & 72 & TTE, TEE & MVR & Surgery & n.a. \\
\hline 6. Benisty et al. & 2000 & Male & 73 & TTE, TEE & MVR $\times 3$, AVR & Surgery & n.a. \\
\hline 7. Weinrich et al. & 2001 & Female & 58 & TEE, CC & MVRx 2 & Surgery & Survived \\
\hline 8. Wasserman et al. & 2002 & Male & 78 & TTE, TEE, & AVR & Surgery & Survived \\
\hline 9. Cabalka et al. & 2005 & Female & 70 & TTE, TEE & $M V R \times 2$ & Percutaneous & Survived \\
\hline 10. Lorber et al. & 2006 & Female & 78 & TTE, CC & MVR & Percutaneous & Survived \\
\hline 11. Ramasubbu et al. & 2006 & Male & 41 & TEE & Aortic root reconstruction & Surgery & Survived \\
\hline 12. Ramasubbu et al. & 2006 & Female & 44 & TEE & Aortic root reconstruction & Conservative & Survived \\
\hline 13. Trehan et al. & 2006 & Male & 22 & TTE, MRI, CC & VSD + sinus valsalva repair & Percutaneous & Survived \\
\hline 14. Martinez et al. & 2007 & Female & 70 & TTE & MVR & Percutaneous & Survived \\
\hline 15. Martinez et al. & 2007 & Male & 67 & TTE & AVR & Percutaneous & Survived \\
\hline 16. Uslu et al. & 2007 & Male & 54 & TTE & MVR & Surgery & Survived \\
\hline 17. Hilberath et al. & 2007 & Male & 68 & TEE & AVR + endocarditis & Surgery & Survived \\
\hline 18. Frigg et al. & 2008 & Female & 77 & TEE, CC & AVR & Surgery & Survived \\
\hline 19. Moaref et al. & 2008 & Female & 51 & TEE & MVR & Surgery & na \\
\hline 20. Aoyagi et al. & 2008 & Female & 71 & TTE, CC & MVR, TV repair & Surgery & Survived \\
\hline 21. Rothman et al. & 2008 & Male & 86 & TTE, CC & MVR & Percutaneous & Survived \\
\hline 22. Hansalia et al. & 2009 & Female & 46 & TTE & AVR & Surgery & Survived \\
\hline 23. Yared et al. & 2009 & Male & 60 & TTE, TTE & AVR+ endocarditis & na & na \\
\hline 24. Gorki et al. & 2009 & Female & 69 & na & AVR + endocarditis & na & na \\
\hline 25. Subramaniam et al. & 2009 & Male & 60 & TEE, CT & AVR & Surgery & Survived \\
\hline 26. Amirghofran et al. & 2009 & Female & 51 & TEE & MVR & Surgery & Survived \\
\hline 27. Silbiger et al. & 2009 & Female & 30 & TTE, CC & VSD repair & Conservative & Survived \\
\hline 28. Cheema et al. & 2009 & Female & 31 & MRI & VSD repair & Conservative & Survived \\
\hline 29. Can et al. & 2009 & Male & 72 & TTE & AV nod ablation & Conservative & Survived \\
\hline 30. Can et al. & 2009 & Male & 68 & Autopsy & AV nod ablation & na & Died \\
\hline 31. Dadkhah et al. & 2009 & Female & 73 & TEE & TV repair & Conservative & Survived \\
\hline 32. Mohapatra et al & 2009 & Female & 22 & TEE & MVR (RF) & Surgery & Survived \\
\hline 33. Sun et al. & 2010 & na & na & na & MVR & Surgery & na \\
\hline 34. Sun et al. & 2010 & na & na & na & MVR & na & na \\
\hline 35. Pursnani et al. & 2010 & Male & 78 & TTE, TEE & AVR & Surgery & Survived \\
\hline 36. Sharma et al. & 2011 & Male & 80 & TTE & AV nod ablation & Conservative & Survived \\
\hline 37. Kumar et al. & 2011 & Female & 59 & TEE & AVR $\times 2+$ endocarditis & Surgery & Survived \\
\hline 38. Zhu et al. & 2012 & Baby & 6 months & TTE, TEE & ASD, VSD repair & Percutaneous & Survived \\
\hline 39. Bochard-Villanueva & 2012 & Male & 63 & TEE, CT & AVR+ endocarditis & Surgery & Survived \\
\hline 40. Vallakati et al. & 2012 & Female & 53 & TTE & AVR & Conservative & Survived \\
\hline 41. Elmistekawy et al. & 2012 & Male & 59 & TEE & AVR & Surgery & Survived \\
\hline 42. Dores et al. & 2012 & Male & 50 & TTE, TEE & AVR, MVR & Surgery & Survived \\
\hline 43. Yurdakul et al. & 2012 & Male & 68 & TEE & AVR & Surgery & Survived \\
\hline 44. Mousavi et al. & 2012 & Female & 76 & TEE, MRI & AVR & Conservative & Survived \\
\hline
\end{tabular}


Table 2 Patients with acquired Gerbode defect undergoing previous cardiac surgery (Continued)

\begin{tabular}{|c|c|c|c|c|c|c|c|}
\hline 45. Ozdogan et al. & 2012 & Female & 31 & TTE, TEE & MVRx2 + endocarditis & Surgery & Died \\
\hline 46. Anderson et al. & 2012 & na & na & na & AVR & na & na \\
\hline 47. Toprak et al. & 2013 & Male & 32 & TTE, TEE & AVR & Conservative & Survived \\
\hline 48. Notarangelo et al. & 2013 & n.a. & 69 & TTE, TEE & MVR & Percutaneous & Survived \\
\hline 49. Sinisalo et al. & 2013 & Male & 75 & TTE, TEE, CC & AVR & Percutaneous & Survived \\
\hline 50. Sinisalo et al. & 2013 & Female & 23 & TEE, CC & VSD repair & Percutaneous & Survived \\
\hline 51. Sinisalo et al. & 2013 & Male & 10 & TEE, CC & ASD, VSD repair & Percutaneous & Survived \\
\hline 52. Sinisalo et al. & 2013 & Male & 8 & TEE, CC & VSD repair & Percutaneous & Survived \\
\hline 53. Dangol et al. & 2013 & Male & 6 months & TTE,TEE,CC & ToF repair & Percutaneous & Survived \\
\hline 54. Lee et al. & 2013 & Male & 3 months & TTE, CC & ASD, PDA, VSD repair & Percutaneous & Survived \\
\hline 55. Poulin et al. & 2013 & Female & 75 & TTE,TEE & MVR & Percutaneous & Survived \\
\hline 56. Primus et al. & 2013 & Female & 76 & TTE,TEE & AVR & Conservative & Survived \\
\hline 57. Chaturvedi et al. & 2013 & Male & 62 & TTE, MRI & AVR & Percutaneous & Survived \\
\hline 58. Tayama et al. & 2014 & Male & 75 & TTE, CC & MV and TV repair & Surgery & Survived \\
\hline 59. Hussain et al. & 2014 & Male & 45 & TTE, TEE & AVRx2 & Surgery & Survived \\
\hline 60. Chamsi-Pasha et al & 2014 & Male & 67 & TTE, TEE & MVR, TVR & Surgery & Survived \\
\hline 61. Taskesen et al. & 2014 & Male & 74 & TTE, TEE & AVR $\times 2$ & Percutaneous & Survived \\
\hline 62. Fanari et al & 2015 & Female & 50 & TTE, CT & AVR & Percutaneous & Survived \\
\hline
\end{tabular}

Patients with acquired Gerbode defect undergoing previous cardiac surgery

Legend: TTE Transthoracic echocardiography, TEE Transesophageal echocardiography, CC Cardiac catheterization, CT Cardiac tomography, MRI Magnetic

resonance, na-not available, AVR Aortic valve replacement, MVR Mitral valve replacement, TV Tricuspid valve, ASD Atrial septal defect, VSD Ventricular septal defect, ToF Tetralogy of Fallot, PDA Patent ductus arteriosum

as transesophageal echocardiography provide excellent images for guidance in device sizing and deployment. The Amplatzer duct occluder device is a mainstay in treatment as it provides less radial force [30] than the muscular ventricular septal defect closure device causing fewer complications [31].

In most of the cases with acquired Gerbode defect a simple direct suture might be enough to close the defect [12] such as in our case, although large Gerbode defect associated with partial or total distruction of the tricuspid valve can be much more challenging. In such cases reconstruction or replacement of the tricuspid valve might be required. Tatewaki et al. [15] describe a pericardial patch closure with sutures from the ventricular side of the tricuspid valve through the leaflets. Others reported a Dacron patch closure with septal leaflet reimplantation onto the patch $[9,12]$, an annuloplasty ring implantation, or tricuspid valve replacement $[5,8,9,12]$. Matt et al. [20] presented a double plicated patch combining a defect closure and reconstruction of the tricuspid valve annulus and septal leaflet. In our case we closed the defect from the right side using two single pledgeted prolene suture and reconstruct the septal and anterior tricuspid valve leaflets using an autologous pericardial patch. This technique allowed us to perform a complex right-sided defect repair with one patch that might be advantageous in an infective situation. Such a technique might allow an extensive reconstruction of the tricuspid valve, if necessary.

As conclusion, the acquired Gerbode defect a rare form of intracardiac shunt, but its incidence has been increasing during the last decades. Increased numbers of invasive and repeat cardiovascular procedures and infective endocarditis have led to this increase in acquired Gerbode defect. Surgical treatment is always feasible with excellent outcome. However the percutanous transcatheter closure remains an excellent option especially in high risk patients.

\section{Conclusion}

The acquired Gerbode defect a rare form of intracardiac shunt, but its incidence has been increasing during the last decades. Increased numbers of invasive and repeat cardiovascular procedures and infective endocarditis have led to this increase in acquired Gerbode defect. Surgical treatment is always feasible with excellent outcome. However the percutanous transcatheter closure remains an excellent option especially in high risk patients.

\section{Consent}

Written informed consent was obtained from the patient for publication of this Case report and any accompanying images. A copy of the written consent is available for review by the Editor-in-Chief of the Journal of Cardiothoracic Surgery. 


\section{Competing interests}

We declare that we do not have any competing or financial interests with this manuscript.

\section{Authors' contribution}

$F A$ and $A D$ diagnosed the patient and followed the patient postoperatively. $E P$ and $A B$ performed the surgery. All authors were involved on the literature review and manuscript writing process. All authors read and approved the final manuscript.

\section{Authors' information}

Edvin Prifti, MD, PhD, Fadil Ademaj, MD, Arben Baboci, MD, Aurel Demiraj, MD.

\section{Author details}

${ }^{1}$ Division of Cardiac Surgery, University Hospital Center of Tirana, Tirana, Albania. ${ }^{2}$ Division of Cardiology, Regional Hospital of Gjakovo, Gjakovo, Kosovo. ${ }^{3}$ Division of Heart Disease, Gjakovo Hospital, Rr. Prizren, Gjakove, Kosove, Albania.

\section{Received: 3 May 2015 Accepted: 24 August 2015}

\section{Published online: 09 September 2015}

\section{References}

1. Thurman J. Aneurysms of the heart. Med Clin Trans R. 1838:21:187.

2. Gerbode F, Hultgren H, Melrose D, Osborn J. Syndrome of left ventricular-right atrial shunt; successful surgical repair of defect in five cases, with observation of bradycardia on closure. Ann Surg. 1958;148:433-46.

3. Cantor S, Sanderson R, Cohn K. Left ventricular-right atrial shunt due to bacterial endocarditis. Chest. 1971:60:552-4.

4. Taskesen T, Prouse AF, Goldberg SL, Gill EA. Gerbode defect: Another nail for the 3D transesophagel echo hammer? Int J Cardiovasc Imaging. 2015;31(4):753-64.

5. Battin M, Fong LV, Monro JL. Gerbode ventricular septal defect following endocarditis. Eur J Cardiothorac Surg. 1991;5(11):613-4.

6. Saiki Y, Kawase M, Ida T, Mannouji E, Kasegawa H, Takahashi Y, et al. The successful surgical repair of a left ventricular- right atrial communication and aneurysm of the mitral valve caused by infective endocarditis: report of a case. Surg Today. 1994;24:655-8.

7. Katoh J, Okabe H, Ujiie T, Edamoto Y, Murakami H, Sakurai M, et al. A case report of left ventricular-right atrial com munication with tricuspid regurgitation due to infective endo- carditis. Jpn J Thorac Surg. 1994;47:228-23.

8. Elian D, Di Segni E, Kaplinsky E, Mohr R, Vered Z. Acquired left ventricular-right atrial communication caused by infective endocarditis detected by transesophageal echocar- diography: case report and review of the literature. J Am Soc Echocardiogr. 1995;8:108-10.

9. Velebit V, Schoneberger A, Ciaroni S, Bloch A, Maurice J, Christenson JT, et al. "Acquired" left ventricular-to-right atrial shunt (Gerbode defect) after bacterial endocarditis. Tex Heart Inst J. 1995:22:100-2.

10. Winslow TM, Friar DA, Larson AW, Barry MJ. A rare complication of aortic valve endocarditis: diagnosis with trans- esophageal echocardiography. J Am Soc Echocardiogr. 1995;8:546-50.

11. Michel C, Rabinovitch MA, Huynh T. Gerbode's defect associated with acute sinus node dysfunction as a complication of infective endocarditis. Heart. 1996;76:379.

12. Alphonso N, Dhital K, Chambers J, Shabbo F. Gerbode's defect resulting from infective endocarditis. Eur J Cardiothorac Surg. 2003;23:844-6.

13. Raja Y, Jenkins N, Chauhan A, Millner RW. Acquired post-infarct Gerbode defect complicated by infective endocarditis with giant right atrial vegetation. Int J Cardiol. 2006;113:E79-80.

14. Fukui K, Kanazawa J, Kawamura T, Yamauchi S, Watanabe K, Taniguchi S, et al. Left ventricular-right atrial communi- cation resulting from infective endocarditis. Jpn J Thorac Surg. 2007;60:213-6.

15. Tatewaki H, Alesnik JP, Morales DSL. Acquired lef ventricle to right atrial shunt (Gerbode defect) and massive pul monary embolus. 2008; http://www.ctsnet.org/sections/clinicalresources/clinicalcases/article-14

16. Inoue $H$, Iguro $Y$, Kinjo T, Matsumoto H, Yotsumoto G, Sakata R. Acquired left ventricular-right atrial communication and severe aortic valve regurgitation caused by infective endocarditis. Thorac Cardiovasc Surg. 2009;57:54-6.
17. Cortez-Dias N, Varela MG, Marques J, Sargento L, Martins S, Ribeiro MA, et al. Acquired left ventricular-to-right atrial shunt (Gerbode defect) due to infective endocarditis. Port J Cardiol. 2009;28:735-9.

18. Mendoza DD, Wang Z, Gaglia MA, Taylor AJ. Gerbode defect. J CardiovasC Comput Tomogr. 2009;3:279-81.

19. Hori D, Tanaka M, Yamaguchi A, Adachi H. Surgically treated infective endocarditis involving the aortic bicuspid valve and ventricular septum revealing aortic regurgitation and a Ger- bode defect. Gen Thorac Cardiovasc Surg. 2010;58:255-9.

20. Matt P, Winkler B, Carre T, Eckstein F. Plicated Patch Repair for Acquired Gerbode Defect Involving the Tricuspid Valve. Ann Thorac Surg. 2010;89:643-5

21. Ota T, Yamaguchi R, Tanigawa T, Otuka K, Hayashi Y, Nishiyama H. Left ventricular-right atrial communication by perforation of the atrioventriculas portion of the membranous septum and severe aortic valve regugitation caused by infective endocarditis. J Echocardiogr. 2011;9:30-2.

22. Pillai V, Menon S, Kottayil B, Karunakaran J. Tricuspid endocarditis with indirect Gerbode: septal translocation of pos- terior leaflet. Heart Lung Circ. 2011;20:362-4.

23. Carpenter RJ, Price GD, Boswell GE, Nayak KR, Ramirez AR. Gerbode defect with Staphylococcus lugdunensis native tricuspid valve infective endocarditis. J Card Surg. 2012;27:316-20.

24. Hsu SY, Shen TC. A spontaneously closed, acquired supravalvular Gerbode defect mimicking an unruptured sinus of Valsalva aneurysm. Eur Heart J Cardiovasc Imaging. 2014;15:471.

25. Hole T, Wiseth $\mathrm{R}$, Levang $\mathrm{O}$. Post-infarction left ventricle to right atrium fistula diagnosed by transthoracic Doppler echocardiography. Eur Heart J. 1995; 16:866-8

26. Jobic Y, Verdun F, Guillo P, Bezon E, Gilard M, Etienne Y, et al. Postinfarction atrioventricular septal rupture. J Am Soc Echocardiogr. 1997;10:680-4.

27. Newman Jr JN, Rozanski L, Kreulen T. Acquired left ventricular to right atrial intracardiac shunt after myocardial in- farction: a case report and review of the literature. J Am Soc Echocardiogr. 1996:9:716-20.

28. Venkatesh G, Lonn EM, Holder DA, Williams WG, Mulji A. Acquired left ventricular to right atrial communication and complete heart block following nonpenetrating cardiac trauma. Can J Cardiol. 1996;12:349-52.

29. Selinger $L$, Werner $K$, Silber $R$, Nellessen $U$, Inselmann G. Natural history of a ventriculoatrial fistula after a gunshot injury in 1945. Ann Thorac Surg. 1998:65:1137-8.

30. Taskesen T, Goldberg SL, Gill EA. Role of three-dimensional echocardiography in management of acquired intracardiacshunts. Echocardiography. 2014;31:E250-3.

31. Fanari Z, Barekatain A, Abraham N, Hopkins JT. Percutaneous closure of acquired Gerbode defect: management of a rare complication of cardiac surgery. Interact Cardiovasc Thorac Surg. 2015;4.

\section{Submit your next manuscript to BioMed Central and take full advantage of:}

- Convenient online submission

- Thorough peer review

- No space constraints or color figure charges

- Immediate publication on acceptance

- Inclusion in PubMed, CAS, Scopus and Google Scholar

- Research which is freely available for redistribution 